\title{
UNBOUNDED VECTOR MEASURES
}

by

William Byers

A thesis submitted to the Faculty of Graduate Studies and Research of McGill University, in partial fulfillment of the requirements for the degree of Master of Science.

Mathematics Department

McGill University 


\section{ACKNOWLEDGMENT}

The author wishes to express his gratitude to Professor A. Evans for the generous gift of his time and ideas during the preparation of this thesis. 
1. Introduction

Chapter 1

2. Preliminary Definitions

3. Main Theorem

5

4. Definition of Unbounded Vector Measures

Chapter 11

5. The Range of a Bounded Non-Atomic Vector Measure

6. The Range of a Non-Atomic Unbounded Vector Measure

7. A Counter-Example 


\section{INTRODUCTION}

The basic aim of this thesis is to extend the definition of an n-dimensional vector measure so as to allow it to assume infinite values. In the l-dimensional case, when one extends the notion of a non-negative measure to that of a signed measure which may assume negative values, it is necessary to assume that the signed measure takes on at most one of the values $(+\infty)$ or $(-\infty)$. In a similar fashion it is shown that in order to successfully extend the definition of a finite-valued n-dimensional measure, it is necessary to suppose that the extended measure assumes at most one infinite value.

In 1940, A. Liapounoff [I] proved that the range of a countably additive finite measure with values in a finitedimensional real vector space is closed and in the non-atomic case, convex. In chapter 1I, it is proved that a strongly $\sigma-f$ inite, non-atomic measure which takes on values in a compactification of Euclidean n-space has a range which is convex but not necessarily closed.

In a recent paper by Gould, [6], there is a discussion of bounded and unbounded vector measures which take on values in Banach spaces. However his generalization to unbounded measures is not the direct generalization of the one-dimensional case that is considered here. In the present discussion we preserve countable additivity over the given $\sigma$-ring, which cannot be done with more general measures. 
CHAPTER 1

\section{Preliminary Definitions}

Let $R$ be the space of real numbers and let $E^{n}$ be Euclidean n-space regarded as a normed n-dimensional vector space. Since al.1 norms on $E^{\text {n }}$ are equivalent, we shall employ the Euclidean norm in all of the following without any loss of generality.

We first construct a completion of $\mathrm{E}^{\mathrm{n}}$. Consider the ma.pping

$$
f: E^{n} \longrightarrow D^{n}=\left\{x \in E^{n}:\|x\| \leqslant I\right\}
$$

given by

$$
f(x)=x /(\|x\|+1)
$$

This is the usual homeomorphism between $\mathrm{E}^{\text {n }}$ and the set

$$
\left\{x \in \mathbb{E}^{n}:\|x\|<1\right\}
$$

which is the interior of the unit disk, $D^{n}$. Since the closure of

$$
\left\{x \in E^{n}:\|x\|<1\right\}
$$

is $D^{\mathrm{n}}$ which is compact, $\mathrm{D}^{\mathrm{n}}$ is a compactification of the space $\mathrm{E}^{\mathrm{n}}$ under the homeomorphism $\mathrm{s}$.

The space $\mathrm{T}$ which we shall be considering is homeomorphic to this compactification of $\mathrm{E}^{\mathrm{n}}$. It can be expressed as the disjoint union, $T=E^{n} \cup S^{n-1}$, where $S^{n-1}$ is the set consisting of a.ll points in $\mathbb{E}^{\mathrm{n}}$ of unit norm. The points of $\mathrm{S}^{\mathrm{n}-1}$ will be identified 
by writing them in the form " $\alpha$ ", where $\alpha$ is a unit vector. The topology defined on $\mathrm{T}$ will be the homeomorphic image under $f$ of the usual topology on $\mathrm{D}^{\mathrm{n}}$. This topology may be fully described by giving a neighbourhood base for each point in T. The base for a point $x \in E^{n}$ is composed of sets of the form

$$
V_{\epsilon}=\left\{y \in E^{n}:\|x-y\|<\epsilon\right\} \text {, for } \epsilon>0 \text {. }
$$

For a point $\alpha^{\infty}$ we take the neighbourhood base given by the sets

$$
V(k, \epsilon)=\left\{x \in E^{n}:\|x\|>k,\|x /\| x\|-\alpha\|<\epsilon\right\}
$$

where $k, \epsilon$ are arbitrary positive numbers. We can easily see that $\mathrm{V}(\mathrm{k}, \epsilon)$ is homeomorphic to the open set

$$
\{x: k /(1+k)<\|x\|<I,\|x /\| x\|-\alpha\|<\varepsilon\}
$$

contained in the interior of $\mathrm{D}^{\mathrm{n}}$.

Thus we can easily extend the concept of convergence in $E^{n}$ to $T$. By the expression " the sequence $\left\{x_{m}\right\}$ converges to $\alpha^{\infty}$, $x_{m} \in E^{n}, m=1,2,3, \ldots$ " which we write as

$$
x_{m} \longrightarrow \alpha^{\infty} \text { as } \mathrm{m} \longrightarrow \infty \text {, }
$$

we mean that for every neighbourhood $V$ of $\alpha^{\infty}$ there exists an integer $N>0$ such that

$$
m>\mathbb{N} \text { implies } x_{m} \in V \text {. }
$$

Now in terms of our basic neighbourhoods this states that for al1 $\mathrm{k}>0$ and any $\epsilon>0$ there exists an integer $N>0$ such that

$$
x_{m} \in V(k, \epsilon) \text { for } a l l \quad m>N,
$$


which implies that

$$
\mathrm{x}_{\mathrm{m}} /\left\|\mathrm{x}_{\mathrm{m}}\right\| \longrightarrow \alpha \text { as } \mathrm{m} \longrightarrow \infty
$$

and

$$
\left\|x_{m}\right\| \longrightarrow \cdots \rightarrow \infty \text { as } m \longrightarrow \infty
$$

Let $X$ be the space on which our set functions are to be defined and let $S$ be a orring of subsets of $X$. Let $u$ be a function defined on the sets of $S$ which takes on values in $T$. We shall always assume that $u$ is countably additive on sets of finite measure; i.e. If $E \in S,\|u(E)\|<\infty$, and if $E=\bigcup_{n=1}^{\infty} E_{n}$ where the sets $E_{n}$ are disjoint and $\left\|\mu\left(E_{n}\right)\right\|<\infty \quad n=1,2,3, \ldots$ then

$$
u(E)=\sum_{n=1}^{\infty} \mu\left(E_{n}\right) \text { or } \lim _{N \rightarrow \infty} \sum_{n=1}^{N} \mu\left(E_{n}\right)=\mu(E) .
$$

We shall also assume that $\mu(\Phi)=0$ where $\Phi$ denotes the empty set and $O$ is the null vector; and that the function $\mu$ is strongly o-finite, i.e. if $E \in S$ then there exists a sequence $\left\{E_{n}\right\}$ of disjoint measurable sets such that $E$ is contained in the union of the $E_{n}$ and $\| \mu\left(E_{n}\right) \mid<\infty \quad n=1,2, \ldots$

\section{Conventions:}

We shall make the following operational definitions for using the symbol $\alpha^{\infty}$ :

$$
\begin{aligned}
& \text { (Ia) } \alpha^{\infty}+\alpha^{\infty}=\alpha^{\infty} \\
& \text { (Ib) } \sum_{n=1}^{\infty}\left(\alpha^{\infty}\right)=\alpha^{\infty} \\
& \text { (2) } \lambda(\alpha)=\alpha^{\infty}, \quad \text { where } \lambda \in R, \lambda>0 \\
& \text { (3) } \alpha^{\infty}+x=\alpha^{\infty}, \quad \text { where } x \in E^{n}
\end{aligned}
$$




\section{Main Theorem}

In this section we extend the definition of the measure of a set to the case where the value of the measure is of the form $\alpha^{\infty}$. We then proceed to show that a measure can assume at most one infinite value.

\section{Definition 3.1}

Let $E \in S$. We say that $\mu(E)=2^{\infty}$ if for every countable disjoint decomposition of $E$ into measurable sets, $E={ }_{n}^{\infty} E_{n}$, satisfying $\left\|\mu\left(E_{n}\right)\right\|<\infty \quad n=1,2, \ldots$ we have:

$$
\mid \sum_{n=1}^{N} u\left(E_{n}\right): \longrightarrow \infty \text { as } N \longrightarrow \infty
$$

and

$$
\sum_{n=1}^{N} \mu\left(E_{n}\right) /\left\|\sum_{n=1}^{N} \mu\left(E_{n}\right)\right\| \longrightarrow \alpha \quad \text { as } \quad N-\cdots \infty
$$

Since $\mu$ is strongly $\sigma$-finite over $S$, each set with measure $2^{\infty}$ has at least one such decomposition. If the limit in (3.2) does not exist for some decomposition, the set function $\mu$ will not be a measure on $\mathrm{S}$.

\section{Lemma 3.2}

Consider a sequence of vectors in $\mathrm{E}^{\mathrm{n}},\left\{\mathrm{x}_{\mathrm{n}}\right\}_{\mathrm{n}=1}^{\infty}$, such that:

$$
\mathrm{x}_{\mathrm{n}} /\left\|\mathrm{x}_{\mathrm{n}}\right\| \longrightarrow \cdots \quad \text { as } \mathrm{n} \longrightarrow \cdots \rightarrow \infty
$$

and

$$
\left\|x_{n}\right\| \longrightarrow \infty \text { as } n \longrightarrow \infty
$$

Let $z$ be a fixed vector.

Then

$$
\text { (a) }\left\|x_{n}+z_{\|}\right\| \longrightarrow \infty
$$




$$
\begin{aligned}
& \text { (b) }\left\|x_{n}\left|/ \| x_{n}+z\right| \longrightarrow 1\right. \\
& \text { (c) }\left(x_{n}+z\right) /\left\|x_{n}+z\right\| \longrightarrow \alpha
\end{aligned}
$$

Proof:

(a) (3.5) follows from (3.4)

(b) $\left|1-\left\|x_{n}\right\| /\left\|x_{n}+z\right\|\right|=\left|\left(|| x_{n}+z\|-\| x_{n} \|\right) /\left\|x_{n}+z\right\|\right|$

$$
\leqslant\|z\| /\left\|x_{n}+z\right\|
$$

which can be made as small as we wish for sufficiently large $n$ since $\left\|x_{n}+z\right\| \longrightarrow \infty$

(c) Let $\epsilon>0$ be given.

From (3.3) there exists $\mathbb{N}_{1}$ such that $\left\|\alpha-x_{n} /\right\| x_{n}\|\|<\epsilon / 3$ whenever $\mathrm{n}>\mathrm{N}_{\mathrm{I}}$,

from (3.6) there exists $\mathbb{N}_{2}$ such that $\mid$ I $-\left\|x_{n}\right\| /\left\|x_{n}+z\right\| \mid<\epsilon / 3$ whenever $\mathrm{n}>\mathrm{N}_{\mathfrak{Z}}$,

from (3.5) there exists $\mathbb{N}_{3}$ such that $\|z\| /\left\|x_{n}+z\right\|<\epsilon / 3$ whenever $\mathrm{n}>\mathbb{N}_{3}$.

Let $\mathbb{N}=\max \left\{\mathbb{N}_{1}, \mathbb{N}_{2}, \mathbb{N}_{3}\right\}$; then

$\left\|\alpha-\left(x_{n}+z\right) /\right\| x_{n}+z\|\|$

$=\left\|\alpha-\left(x_{n} /\left\|x_{n}\right\|\right)+\left(x_{n} /\left\|x_{n}\right\|\right)-\left(x_{n} /\left\|x_{n}+z\right\|\right)+\left(x_{n} /\left\|x_{n}+z\right\|\right)-\left[\left(x_{n}+z\right) /\left\|x_{n}+z\right\|\right]\right\|$

$\leqslant\left\|\alpha-x_{n} /\right\| x_{n}\|\|+\left\|x_{n}\right\|\left(\left|l /\left\|x_{n}\right\|-1 /\left\|x_{n}+z\right\|\right|\right)+\|z\| /\left\|x_{n}+z\right\|$

$=\left\|\alpha-x_{n} /\right\| x_{n}\|\|+\|I-\| x_{n}\|/\| x_{n}+z\|\mid+\| z\|/\| x_{n}+z \|$

$\langle\epsilon / 3+\epsilon / 3+\epsilon / 3=\epsilon$ for $n>\mathbb{N}$

Thus $\left(x_{n}+z\right) /\left\|x_{n}+z\right\| \longrightarrow \alpha$ as $n \longrightarrow \infty$.

Theorem 3.3

Suppose $E$ and $F$ are two disjoint measurable sets such that $u(E)=\alpha, \mu(F)=\beta \infty, \quad(\alpha \neq \beta)$. Then there exists a decomposition of $E U F$ into a countable union of disjoint measurable sets of finite 
measure such that the limit in (3.2) does not exist and therefore $u$ is not a measure on $\mathrm{S}$.

Proof:

Since $u(E)=\alpha^{\infty}$ and $u$ is strongly $\sigma$-finite, there exists a decomposition of $E$ into disjoint measurable sets, $E=\bigcup_{n=1}^{\infty} E_{n}$, such that $\left\|u\left(E_{n}\right)\right\|<\infty \quad n=1,2,3, \ldots$ and the sequence $\left\{E_{n}\right\}_{n=1}^{\infty}$ satisfies (3.1) and (3.2). Similarly, $F=\bigcup_{n=1}^{\infty} F_{n}$ is such a decomposition of F.

Consider the sequence of partial sums, $\left\{\sum_{i=\mathbb{N}}^{k} \mu\left(E_{i}\right)\right\}_{k=N}^{\infty}$,

where $\mathrm{N}$ is any fixed positive integer. In the hypothesis of lemma (3.2), put

$$
x_{k}=\sum_{i=1}^{k} u\left(E_{i}\right) \text { and } z=-\sum_{i=1}^{N-1} u\left(E_{i}\right)
$$

Then since $\left\{E_{n}\right\}_{n}$ satisfies (3.1) and (3.2) it follows that $\left\{x_{k}\right\}_{k=1}^{\infty}$ satisfies (3.3) and (3.4). Since

$$
\|z\| \leqslant \sum_{i=1}^{N-1}\left\|u\left(E_{i}\right)\right\|<\infty
$$

it follows from the conclusions of lemma 3.2 that

$$
\left\|\sum_{-}^{k} \mu\left(E_{i}\right)\right\| \longrightarrow \ldots \text { as } k \longrightarrow \infty
$$

and

$$
\sum_{i=\mathbb{N}}^{k} u\left(E_{i}\right) /\left\|\sum_{i=\mathbb{N}}^{k} u\left(E_{i}\right)\right\| \longrightarrow \infty \text { as } k \longrightarrow \infty
$$

Obviously the same result holds for $F=\bigcup_{n=1}^{\infty} F_{n}$.

Let $\mathrm{z}$ be an arbitrary vector in $\mathrm{E}^{\mathrm{n}}$. In the hypothesis of lemma 3.2 , let

$$
\mathrm{x}_{\mathrm{k}}=\sum_{i=\mathbb{N}}^{k} \mu\left(E_{i}\right)
$$


From (3.8) and (3.9) it follows that $\left\{x_{k}\right\}_{k=N}^{\infty}$ satisfies the hypothesis of lemma 3.2 and therefore

$$
\left[z+\sum_{i=\mathbb{N}}^{k} u\left(E_{i}\right)\right\rceil /\left\|z+\sum_{i=\mathbb{N}}^{k} u\left(E_{i}\right)\right\| \cdots \rightarrow \infty \text { as } k-\cdots \rightarrow \infty
$$

and

$$
\left\|z+\sum_{i=N}^{k} \mu\left(E_{i}\right)\right\| \longrightarrow \infty \text { as } k \longrightarrow \infty
$$

Again the same result holds for $F=\bigcup_{n=1}^{\infty} F_{n}$.

Suppose $\|\alpha-\beta\|=\delta>0$.

Select $\epsilon$ such that $0<\epsilon<\varepsilon / 2$.

Since

$$
\sum_{i=1}^{n} u\left(E_{i}\right) /\left\|\sum_{i=1}^{n} u\left(E_{i}\right)\right\| \longrightarrow \alpha \text { as } n \longrightarrow \infty
$$

there exists $N$ such that

$$
\left\|\alpha-\sum_{i=1}^{N} u\left(E_{i}\right) /\right\| \sum_{i=1}^{N} u\left(E_{i}\right)\|\|<\epsilon .
$$

Let $n_{1}$ be the first such $N$.

$$
\text { Let } z=\sum_{i=1}^{n_{1}} u\left(E_{i}\right) \text {. Then }\|z\| \leqslant \sum_{i=1}^{n_{1}}\left\|u\left(E_{i}\right)\right\|<\infty \text {. Thus it }
$$

follows from the analogue of (3.10) for $F=\bigcup_{n=1}^{\infty} F_{n}$ that there exists $M$ such that

$$
\left\|\beta-\left[\sum_{i=1}^{n_{1}} u\left(E_{i}\right)+\sum_{i=1}^{M} u\left(F_{i}\right)\right] /\right\| \sum_{i=1}^{n_{1}} u\left(E_{i}\right)+\sum_{i=1}^{M} u\left(F_{i}\right)\|\|<\epsilon .
$$

Let $m_{1}$ be the first such $M$.

Suppose we have picked $n_{1}, \ldots, n_{k-1} ; m_{1}, \ldots, m_{k-1}$. If $z=\sum_{i=1}^{m_{k-1}} \mu\left(F_{i}\right)$

then (3.10) implies that there exists $N>n_{k-1}$ such that 


$$
\left\|\alpha-\left[\sum_{i=1}^{m_{k-1}} u\left(F_{i}\right)+\sum_{i=1}^{N} u\left(E_{i}\right)\right] /\right\| \sum_{i=1}^{m_{k}-1} u\left(F_{i}\right)+\sum_{i=1}^{N} u\left(E_{i}\right)\|\|<\epsilon
$$

Iet $n_{k}$ be the first such $N$. Similarly we pick $m_{k}$.

Thus by this inductive process we have arranged the two

sequences $\left\{E_{n}\right\}_{n=I}^{\infty}$ and $\left\{F_{n}\right\}_{n=I}^{\infty}$ into a single sequence:

$$
E_{1}, \ldots, E_{n_{1}}, F_{1}, \ldots, F_{m_{1}}, E_{n_{1}}+1, \ldots, E_{n_{2}}, F_{m_{1}}+1, \ldots, F_{m_{2}}, \ldots
$$

Rename these sets $\mathrm{H}_{1}, \mathrm{H}_{2}, \mathrm{H}_{3}, \ldots$ while preserving the above order. Then $\left\{H_{n}\right\}_{n=I}^{\infty}$ is a sequence of disjoint sets of finite measure whose union is $(E \cup F)$.

We first show that $(E \cup F)$ cannot have finite measure. From (3.5) of lemma 3.2 it follows that there exists an integer $t_{1}$ such that

$$
\left\|u\left(F_{1}\right)+\sum_{i=1}^{t_{1}} u\left(E_{i}\right)\right\|>1
$$

Given $t_{1}, \ldots, t_{k-1}$ there exists $t_{k}>t_{k-1}$ such that

$$
\left\|\sum_{i=1}^{k} u\left(F_{i}\right)+\sum_{i=1}^{t_{k}} u\left(E_{i}\right)\right\|>k
$$

Thus by induction we have a sequence of sets

$$
F_{1}, E_{1}, \ldots, E_{t_{1}}, F_{2}, E_{t_{1}+1}, \ldots, E_{t_{2}}, F_{3}, \ldots
$$

which are measurable, disjoint, of finite measure, and whose union is $(E \cup F)$. If $(E \cup F)$ had finite measure, any such decomposition of $(E \cup F)$ would necessarily be such that the norms of the sequence of partial sums would be bounded. (This follows from the countable additivity of $u$ on $S$ and the definition of convergence in our space.) Since this is not so for the decomposition we have developed, ( $E \cup F$ ) cannot have finite measure.

Suppose $u(E \cup F)=\gamma^{\infty}$, for some $\gamma$. Because of definition 3.1 
our decomposition $\bigcup_{n=1}^{\infty} H_{n}=E \cup F$ must satisfy condition (3.2)

i.e. $\sum_{k=1}^{N} \mu\left(H_{k}\right) /\left\|\sum_{k=1}^{N} \mu\left(H_{k}\right)\right\|_{1} \longrightarrow \gamma$ as $\mathbb{N} \longrightarrow \infty$

Now if $\|\alpha-\gamma\| \leqslant \epsilon$, we must have

$$
\begin{aligned}
\|\beta-\gamma\|=\|(\beta-\alpha)-(\gamma-\alpha)\| & \geqslant\|\beta-\alpha\|-\|\gamma-\alpha\| \\
& \geqslant \delta-\epsilon>2 \epsilon-\epsilon=\epsilon
\end{aligned}
$$

Thus either $\|\alpha-\gamma\|>\epsilon$ or $\|\beta-\gamma\|>\epsilon$, Without loss of generality we may suppose that $\|\alpha-\gamma\|=t\rangle \epsilon$. Choose $\lambda$ such that $0<\lambda<t-\epsilon$.

Now (3.12) implies that there exists $\mathbb{N}$ such that

$$
\left\|y-\sum_{k=1}^{n} u\left(H_{k}\right) /\right\| \sum_{k=1}^{n} \mu\left(H_{k}\right) \|<\lambda \text { for } n>N .
$$

Therefore

$$
\begin{aligned}
\left\|\alpha-\sum_{k=1}^{n} u\left(H_{k}\right) /\right\| \sum_{k=1}^{n} u\left(H_{k}\right)\|\| & =\left\|(\alpha-\gamma)-\left[\sum_{k=1}^{n} u\left(H_{k}\right) /\left\|\sum_{k=1}^{n} u\left(H_{k}\right)\right\|-\gamma\right]\right\| \\
& \geqslant\|\alpha-\gamma\|-\left\|\sum_{k=1}^{n} u\left(H_{k}\right) /\right\| \sum_{k=1}^{n} u\left(H_{k}\right)\|-\gamma\| \\
& >t-\lambda>\in \text { for } n>\mathbb{N}
\end{aligned}
$$

However from our construction of the sequence $\left\{\mathrm{H}_{n}\right\}_{n=1}^{\infty}$ there exists $n_{k}>N$ such that

$$
\begin{aligned}
& \left\|\alpha-\left[\sum_{i=1}^{n_{k}} u\left(E_{i}\right)+\sum_{i=1}^{m_{k-1}} u\left(F_{i}\right)\right] /\right\| \sum_{i=1}^{n_{k}} u\left(E_{i}\right)+\sum_{i=1}^{m_{k-1}} u\left(F_{i}\right)\|\|<\epsilon \\
& \text { i.e. } \quad\left\|\alpha-\sum_{i=1}^{s} u\left(H_{i}\right) /\right\| \sum_{i=1}^{s} u\left(H_{i}\right)\|\|<\epsilon
\end{aligned}
$$

where $H_{s}$ corresponds to $E_{n_{k}}$ in the construction of $\left\{\mathrm{H}_{n}\right\}_{n=1}^{\infty}$ and $s>\mathbb{N}$.

The inequalities (3.13) and (3.14) contradict each other

and therefore $\sum_{k=1}^{n} u\left(H_{k}\right) /\left\|\sum_{k=1}^{n} u\left(H_{k}\right)\right\|$ cannot tend to a limit as 
$n$ tends to infinity; that is, $u(E \cup F) \neq$ yo for any $\gamma$. Thus we. cannot define the measure of $(E \cup F)$ in such a way as to be consistent with our previous definitions and therefore $u$ is not a measure on the $\sigma$-ring $\mathrm{S}$.

Thus we have shown that in order for our set function $u$ to be defined on all sets of a $\sigma$-ring $s$, it is necessary that $u$ not take on distinct infinite values at disjoint measurable sets. Therefore we shall subsequently eliminate this situation from our considerations and assume that non-finite disjoint measurable sets have identical measures.

Lemma 3.4

Suppose $E$ and $F$ are measurable sets such that $E$ is contained in $F$. Then $\|u(F)\|<\infty$ implies $\|\mu(E)\|<\infty$. Proof:

Suppose $u(\mathbb{E})=y^{\infty}$, for some unit vector $\gamma$. Then there exists a decomposition of $E, E=\bigcup_{n=1}^{\infty} E_{n}$, into a countable number of disjoint measurable sets of finite measure such that the sequence $\left\{E_{n}\right\}_{n=1}^{\infty}$ satisfies (3.1) and (3.2) (where " $\alpha$ " is replaced by " $y$ "). Consider $(F-E)$, a measurable set. Since $(F-E) \cap E=\Phi$, it follows from the assumptions made above that either $\|u(F-E)\|<\infty$ or $h(F-E)=y^{\infty}$. In either case there exists a decomposition of $(F-E), F-E=\bigcup_{n=1}^{\infty} F_{n}$, into a countable number of disjoint measurable sets of finite measure. ( In the case $\|u(F-E)\|<\infty$ we take $F_{1}=F-E$ and $\left.F_{i}=\Phi \quad i=2,3, \ldots\right)$

Following the same procedure as that used in the proof of Theorem 3.3, we can find a sequence $\left\{n_{k}\right\}_{k=1}^{\infty}$ of positive integers 
such that

$$
\begin{aligned}
& \text { (i) } n_{1}<n_{2}<\ldots<n_{k}<\ldots \\
& \text { (ii) }\left\|\sum_{i=1}^{k} u\left(F_{i}\right)+\sum_{i=1}^{n_{k}} u\left(E_{i}\right)\right\|>k \quad k=1,2, \ldots
\end{aligned}
$$

Thus we can define the sequence:

$$
\mathrm{F}_{1}, \mathrm{E}_{1}, \ldots, \mathrm{E}_{\mathrm{n}_{1}}, \mathrm{~F}_{2}, \mathrm{E}_{\mathrm{n}_{1}+1}, \ldots, \mathrm{E}_{\mathrm{n}_{2}}, \ldots
$$

Evidently the sequence is composed of disjoint, measurable sets of finite measure whose union is the set $F$.

Since $F$ is of finite measure, the partial sums of the sequence of measures of the sets in (3.15) must be bounded in norm. However, they are obviously not by the method of construction of the sequence.

\section{This contradiction proves that $\|\mu(E)\|<\infty$.}

Lemma 3.5

Let $\mathrm{E}$ and $\mathrm{F}$ be two disjoint, measurable sets such that $u(E)=u(F)=\alpha^{\infty}$. Then $u(E, J F)=\alpha^{\infty}$.

Proof:

By lemma 3.4,,$(E \cup F)$ cannot be finite.

Suppose $E \cup F=\bigcup_{i=1}^{\infty} H_{i}$ is any decomposition of ( $E \cup F$ ) into disjoint, measurable sets of finite measure. Let $E_{i}=E \cap H_{i}$ and $F_{i}=F \cap H_{i} \quad i=1,2, \ldots$. Then $\left\{E_{i}\right\}_{i=1}^{\infty}$ is a sequence of disjoint, measurable sets such that:

(i) $\bigcup_{i=1}^{\infty} E_{i}=\underset{I}{Y}\left(E \cap H_{i}\right)=E \cap\left(\bigcup_{i} H_{i}\right)=E$

(ii) since $E_{i} \subset H_{i},\left\|\mu\left(H_{i}\right)\right\|<\infty$ implies that $\left\|\mu\left(E_{i}\right)\right\|<\infty$ $i=1,2, \ldots$ (by lemma 3.4). We can make analagous statements about $\left\{F_{i}\right\}_{i=1}^{\infty}$. Therefore, since $u(E)=M(F)=\alpha$, Definition 3.1 implies that the sequences $\left\{E_{i}\right\}_{i=1}^{\infty}$ and $\left\{F_{i}\right\}_{i=1}^{\infty}$ must both satisfy the conditions 
(3.1) and (3.2). We shall now show that $\left\{\mathrm{H}_{i}\right\}_{i=1}^{\infty}$ saitisfies (3.1) and (3.2) which will complete the proof of the lemma. Let $\epsilon>0$ be given.

Let $\mathrm{C}$ be the $\epsilon$-cone about the direction vector $\alpha$; that is, $\mathrm{C}$ is the cone based at the origin each of whose generators makes an angle of $\in$ with the vector $\alpha$. Since $C$ is a cone

$$
\mathrm{x} \in \mathrm{C} \text { implies } \lambda \mathrm{x} \in \mathrm{C} \text { for } a l l \mathrm{x} \in \mathrm{E}^{\mathrm{n}}, \quad \lambda>0
$$

Also $\mathrm{C}$ is convex, therefore

$$
\mathrm{x}, \mathrm{y} \in \mathrm{C} \text { implies } \lambda \mathrm{x}+\pi \mathrm{y} \in \mathrm{C} \text {; where } \lambda+\pi=1, \lambda, \pi>0
$$

Since $\left\{E_{i}\right\}_{i=1}^{\infty}$ and $\left\{F_{i}\right\}_{i=1}^{\infty}$ both satisy condition (3.2), there exists an integer $N$ such that

$n \geqslant N$ implies that $\sum_{i=1}^{n} u\left(E_{i}\right) /\left\|\sum_{i=1}^{n} u\left(E_{i}\right)\right\| \in C$ and $\sum_{i=1}^{n} \mu\left(F_{i}\right) /\left\|\sum_{i=1}^{n} u\left(F_{i}\right)\right\| \in C$. Multiplying by $\left\|\sum_{i=1}^{n} l\left(F_{i}\right)\right\|$ and $\left\|\sum_{i=1}^{n}\left(F_{i}\right)\right\|$ respectively, and using ( 3.16$)$ gives

$$
\sum_{i=1}^{n} u\left(E_{i}\right) \in C \quad \text { and } \quad \sum_{i=1}^{n} u\left(F_{i}\right) \in C, \text { for } n \geqslant N \text {. }
$$

Now (3.17) implies that

$$
(1 / 2) \sum_{i=1}^{n} \mu\left(E_{i}\right)+(I / 2) \sum_{i=1}^{n} u\left(F_{i}\right) \in C, \quad \text { for } n \geqslant N \text {. }
$$

Multiplying by $2 /\left\|\sum_{i=1}^{n} u\left(E_{i}\right)+\sum_{i=1}^{n} u\left(F_{i}\right)\right\|$ and using (3.16)

we have $\left[\sum_{i=1}^{n} u\left(E_{i}\right)+\sum_{i=1}^{n} u\left(F_{i}\right) \pi /\left\|\sum_{i=1}^{n} u\left(E_{i}\right)+\sum_{i=1}^{n} \mu\left(F_{i}\right)\right\| \in C\right.$ for $n \geqslant N$.

Now $u\left(H_{i}\right)=u\left(E_{i} \cup F_{i}\right)=u\left(E_{i}\right)+u\left(F_{i}\right)$, since $E_{i}$ and $F_{i}$ are disjoint 
sets of finite measure. Thus we have shown that

$$
\sum_{i=1}^{n} u\left(H_{i}\right) / \sum_{i=1}^{n} \mu\left(H_{i}\right) \| \in C \text { for } n \geqslant N \text {. }
$$

Since this is true for all $\epsilon>0$, we have

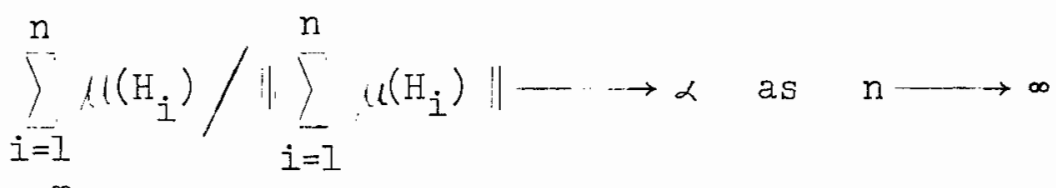

i.e. $\quad\left\{\mathrm{H}_{i}\right\}_{i=1}^{\infty}$ satisfies condition (3.2)

$$
\text { Let } u_{n}=\sum_{i=1}^{n} u\left(H_{i}\right), \quad v_{n}=\sum_{i=1}^{n} u\left(E_{i}\right), w_{n}=\sum_{i=1}^{n} u\left(F_{i}\right) ; n=1,2, \ldots
$$

Since $u_{n}=v_{n}+w_{n}, u_{n}$ lies in the plane determined by the vectors $v_{n}$ and $w_{n}$. Let the angle between $u_{n}$ and $v_{n}$ be $\theta_{1}^{n}$, and that between $u_{n}$ and $w_{n}$ be $\epsilon_{2}^{n}$. Let $\epsilon>0$ be given such that $0<\epsilon<\pi / 3$.

$$
\text { Now } v_{n} /\left\|v_{n}\right\| \longrightarrow \alpha, \quad w_{n} /\left|w_{n}\right| \cdots \rightarrow \alpha \quad \text { as } \quad n \rightarrow \infty \text {; }
$$

and by part $(a), u_{n} /\left\|u_{n}\right\|_{i} \longrightarrow \cdots \rightarrow \alpha$ as $n \rightarrow \infty$. Thus there exists an integer $N>0$ such that $n \geqslant N$ implies $0 \leqslant e_{i}^{n} \leqslant \epsilon \quad i=1,2$. Then ||$u_{n}|=| \mid v_{n}\left\|\cos \theta_{1}^{n}+\right\| w_{n} \| \cos \theta_{2}^{n}$

$$
\begin{aligned}
& \geqslant\left(\left\|v_{n}\right\|+\left\|w_{n}\right\|\right) \cos \epsilon, \quad \text { since } \cos \theta \text { increases as } \theta \text { decreases to } 0 \\
& \geqslant\left(\left\|v_{n}\right\|+\left\|w_{n}\right\|\right)(I / 2), \quad \text { since } 0<\epsilon<\pi / 3 \text { implies } \cos \epsilon>1 / 2
\end{aligned}
$$

This is true for all $n \geqslant N$, and since $\left\|v_{n}\right\|-\rightarrow \infty$ and $\left|w_{n}\right|-\rightarrow \infty$ as $\mathrm{n} \longrightarrow \infty$, we have

$$
\left\|\sum_{i=1}^{n} u\left(H_{i}\right)\right\|=\left\|u_{n}\right\| \longrightarrow \infty \text { as } n \longrightarrow \infty .
$$

Thus $\left\{\mathrm{H}_{i}\right\}_{i=1}^{\infty}$ satisfies condition (3.I) and therefore $u(E \cup F)=\alpha^{\infty}$.

Lemma 3.6

Suppose $E$ and $F$ are two disjcint, measurable sets such that

$u(E)=\alpha^{\infty}$ and $\|u(F)\|<\infty$. Then $u(E \cup F)=\alpha^{\infty}$.

Proof:

If $\|U(E \cup F)\|<\infty$, then, by Iemma 3.4, $\|u(E)\|<\infty$. 
Therefore we must have $u(E \cup F)=\gamma^{\infty}$, for some $\gamma$. Suppose $\gamma \neq \alpha$. Let $E=\bigcup_{n=1}^{\infty} E_{n}$ be any decomposition of $E$ into disjoint measurable sets of finite measure. Let $G_{1}=E_{1} \cup F$. Then

$$
\left\|u\left(G_{1}\right)\right\|=\left\|u\left(E_{1}\right)+u(F)\right\| \leqslant\left\|u\left(E_{1}\right)\right\|+\|, u(F)\|<\infty
$$

Also let $G_{n}=E_{n}$ for $n=2,3, \ldots$. Then $\bigcup_{n=1}^{\infty} G_{n}=E \cup F$ is a decomposition of $(E \cup F)$ into a countable number of disjoint, measurable sets of finite measure.

Since $u(E \cup F)=y^{\infty}$ by assumption, according to definition 3.1 we must have:

$$
\text { (i) }\left\|\sum_{i=1}^{n} u\left(G_{i}\right)\right\|-\rightarrow \text { as } n-\cdots \rightarrow \infty
$$

and

$$
\sum_{i=1}^{n} u\left(G_{i}\right) /\left\|\sum_{i=1}^{n} u\left(G_{i}\right)\right\| \longrightarrow \gamma \text { as } n \longrightarrow \infty
$$

that is,

$$
\left(i^{\circ}\right)\left\|u(F)+\sum_{i=1}^{n} u\left(E_{i}\right)\right\| \longrightarrow \infty \text { as } n \longrightarrow \infty
$$

and

$$
\left(i i^{\circ}\right)\left[u(F)+\sum_{i=1}^{n} u\left(E_{i}\right)\right] /\left\|u(F)+\sum_{i=1}^{n} u\left(E_{i}\right)\right\| \longrightarrow \gamma \text { as } n \longrightarrow \infty
$$

Now, lemma 3.2 implies that

and

$$
\text { (iii) }\left\|\sum_{i=1}^{n} u\left(E_{i}\right)\right\| \longrightarrow \rightarrow \text { as } n \longrightarrow \infty
$$

$$
\text { (iv) } \sum_{i=1}^{n} u^{\prime}\left(E_{i}\right) /\left\|\sum_{i=1}^{n} u\left(E_{i}\right)\right\| \longrightarrow \gamma \text { as } n \longrightarrow \infty
$$

This contradicts the fact that $u(E)=\alpha^{\infty}$. Therefore $u(E \cup F)=\alpha^{\infty}$. 
Corollary to Theorem 3.3

If $E$ and $F$ are measurable sets such that $\mu(E)=\alpha^{\infty}$ and $u(F)=\beta \infty$, where $\alpha \neq \beta$, then $u$ is not a measure on the $\sigma$-ring $S$. Proof:

We consider all possibilities for the measures of the disjoint sets $E \cap F, E-F$, and $F-E$; where $\mu(E)=\alpha^{\infty}$ and $\mu(F)=\beta \infty$, $\alpha \neq \beta$.

(a) Suppose $\|u(E \cap F)\|<\infty$, if

(i) $\|\mu(E-F)\|<\infty$, then $\|\mu(E)\|=\|\mu(E \cap F)+\mu(E-F)\| \leqslant\|u(E \cap F)\|+\|\mu(E-F)\|<\infty$, which gives a contradiction. Therefore $(E-F)$ must have infinite measure and similarly we can show that $(F-E)$ has infinite measure.

(ii) $u(E-F)=y^{\infty}$, for some $\gamma$, then lemma 3.6 implies that $h(E)=y^{\infty}$. Therefore $y=\alpha$. However the same reasoning applied to $F$ gives the result that $\mu(F-E)=\beta \infty$. Thus $(E-F)$ and $(F-E)$ are disjoint, measurable sets with different infinite measures and Theorem 3.3 then implies that $u$ is not a measure on $\mathrm{S}$.

(b) Suppose $\mu(E \cap F)=\alpha^{\infty}$. If $\|\mu(F-E)\|<\infty$ then $u(F)=\alpha_{\infty}$ by lemma 3.6 . This is a contradiction. Therefore $u(F-E)=y^{\infty}$ for some $\gamma$. If $\gamma=\alpha$, then $u(E \cap F)=\lfloor(F-E)=\alpha \infty$ and from lemma $3.5 \mathrm{k}(\mathrm{F})=\alpha \infty$, giving a contradiction. Thus $\gamma \neq \alpha$ and therefore $(F-E)$ and $E$ are disjoint sets with different infinite measures. Therefore Theorem 3.3 implies that $u$ is not a measure on $S$. Similarly $u(E \cap F)=\beta_{\infty}$ implies that $u$ is not a measure on $\mathrm{S}$.

(c) Suppose $u(E \cap F)=y^{\infty}, \gamma \neq \alpha, \beta$. If $\|u(E-F)\|<\infty$, then 
$U(E)=y^{\infty}$ by lemma 3.6 , giving a contradiction. Similarly $\|\mu(F-E)\|=\infty$. If $u(E-F)=u(F-E)=y \infty$, then by lemma 3.5 $\mu(E)=\gamma^{\infty}=\mu(F)$, giving a contradiction. However if $\mu(E-F)=\delta^{\infty}$,

$\delta \neq \gamma$, then we have two disjoint sets, $(E \cap F)$ and $(E-F)$, with different infinite measures and therefore $u$ is not a measure on S. Similarly $u(F-E)=\delta \infty, \delta \neq y$, implies that $\mu$ is not measure on $\mathrm{S}$.

Thus the existence of two measurable sets with difierent infinite measures always leads to the existence of two disjoint sets with different infinite measures which according to Theorem 3.3, implies that $u$ is not a measure on $S$. This situation motivates the definitions of the following paragraph.

4. Definition of Unbounded Vector Measures

In the previous paragraph we showed that to produce a consistent definition of an unbounded vector measure on a o-ring $S$, the range of the measure must contain only one infinite point. We shall call a set function $u$,defined on a oring $S$ with values in the space $T$, which is countably additive on sets of finite measure, strongly $\sigma$-finite, and which assumes one and only one infinite value (in the sense of definition 3.1) an unbounded vector measure. We shall usually denote its unique value by " coll . In the following we show that our unbounded vector measure is a legitimate extension of the usual finite-valued vector measure. 
Iemma 4.1

It follows from the definition of an unbounded vector measure that when $\mu$ is an unbounded vector measure lemmas 3.5 and 3.6 can be extended to the case where the two sets are not necessarily disjoint.

Theorem 4.2

Let $\mu$ be a strongly $\sigma$-finite set function, defined on the o-ring $S$, which assumes one and only one infinite value, namely $\alpha^{\infty}$. If $u$ is countably additive on sets of finite measure, then it is countably additive on all the sets of $S$. Thus an unbounded vector measure preserves the usual properties of a measure.

Proof:

Let $E=\bigcup_{n=1}^{\infty} E_{n}$, where $E, E_{n} \in S \quad n=1,2,3, \ldots$ and $E_{i} \cap E_{j}=\Phi, i \neq j$.

Let $\left\{E_{n_{k}}\right\}_{k}$ be the subsequence of $\left\{E_{n}\right\}_{n}$ consisting of all sets of finite measure and put $F=\mathrm{K}_{\mathrm{nk}}$.

Let $\left\{E_{m_{t}}\right\}_{t}$ be the subsequence of $\left\{E_{n}\right\}_{n}$ consisting of all sets of measure do and put $G=t_{t} E_{m_{t}}$. (Note that these subsequences may in general be finite sequences.) Then $E=F \cup G$.

Suppose $\|U(F)\|<\infty$. Since $"$ is countably additive on sets of finite measure we have

$$
\begin{gathered}
\mu(F)=\sum_{k} u\left(E_{n_{k}}\right) \\
\text { If } u(F)=\alpha^{\infty} \text {, then } F=\bigcup_{k} E_{n_{k}} \text { is a decomposition of } F
\end{gathered}
$$

into a sequence of disjoint measurable sets of finite measure. Therefore

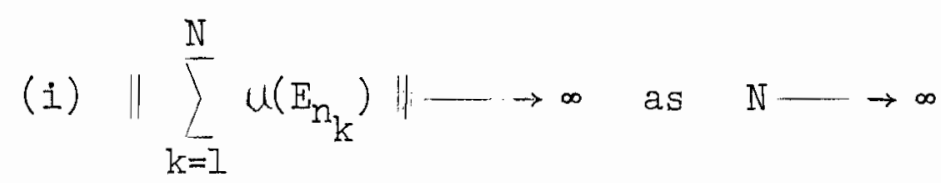


(ii) $\sum_{k=1}^{N} u\left(E_{n_{k}}\right) /\left\|\sum_{k=1}^{N} u\left(E_{n_{k}}\right)\right\| \longrightarrow \alpha$ as $N \longrightarrow \infty$

Thus from the definition of convergence in our space $T$ we have

$$
\begin{gathered}
\mu(F)=\alpha^{\infty}=\sum_{k} \mu\left(E_{n_{k}}\right) \\
\text { If } F=E(\text { i.e. } G=\Phi) \text { then }(4 \cdot I a) \text { and }(4 \cdot I b) \text { prove }
\end{gathered}
$$

the desired result. Now assume $F \neq E$. Then there exists at least

one " $t$ " such that $E_{m_{t}} \subset G \subset \mathbb{E}$. Lemma 3.4 now implies that

$$
u(G)=\alpha=u(E)
$$

Now $u(F)$ is either finite or equal to $\alpha^{\infty}$. In either case

$$
u(G)+u(F)=\alpha \infty+u(F)=\alpha^{\infty}
$$

Moreover

$$
u(G)=\alpha=\sum_{\bar{t}}(\alpha)=\sum_{t} u\left(E_{m_{t}}\right)
$$

(Both (4.3) and (4.4) make use of the operational definitions adopted in paragraph 2.)

$$
\begin{aligned}
& \text { Using (4.1), (4.2), (4.3), and (4.4) we get } \\
& u(E)=\alpha^{\infty}=u(F)+u_{k}(G)=\sum_{k} u\left(E_{n_{k}}\right)+\sum_{t} u\left(E_{m_{t}}\right)=\sum_{n} u\left(E_{n}\right)
\end{aligned}
$$

which proves the theorem. 


\section{The Range of a Bounded Non-Atomic Vector Measure}

In the following section we shall consider Halmos' proof

[2] of the fact that the range of a non-atomic bounded vector measure

is convex. In paragraph six we extend these results to the case of unbounded vector measures.

Definition 5.1

A subset $A$ of $E^{n}$ is said to be convex if $\alpha, \beta \in A$ imply $\lambda \alpha+(1-\lambda) \beta \in A$ for any $\lambda, 0 \leqslant \lambda \leqslant 1$. (We shall later extend this definition to $\mathrm{T}$, the range space of our unbounded vector measures.)

Let $S$ be a $\sigma$-ring of subsets of an arbitrary space $X$ and let $u$ be a bounded, countably additive function of the sets of $S$ with values in $E^{n}$. Then $u$ can be expressed in the form

$$
\mu=\left(\mu_{1}, \ldots, u_{n}\right) \text {, }
$$

an n-tuple of signed measures.

Throughout this paragraph we shall take "measure" to mean

"bounded vector measure" in the above sense, unless otherwise stated.

Definition 5.2

The measure $\mu=\left(\mu_{1}, \ldots, \mu_{n}\right)$ is said to be non-negative if $\mu_{i}(E) \geqslant 0$ for all $E \in S$ and $i=1, \ldots, n$.

Definition 5.3

If $u_{0}$ is a one-dimensional measure, the total varlation, $\mu_{0}^{*}(E)$, of $\mu_{0}$ on the set $E \in S$ is defined by: 


$$
u_{0}^{*}(E)=u_{0}^{+}(E)+u_{d}^{-}(E)
$$

where

$$
\mu_{0}^{+}(E)=\sup \left\{\mu_{0}(F): E \supset F \in S\right\}
$$

and

$$
\mu_{0}(E)=-\inf \left\{\mu_{0}(F): E \supset F \in S\right\}
$$

In general if $\mu=\left(\mu_{1}, \ldots, u_{n}\right), u^{*}$ will denote the non-negative measure $\left(\mu_{1}^{*}, \ldots, u_{n}^{*}\right)$.

\section{Definition 5.4}

The length $\left|u^{*}\right|=\mu_{1}^{*}+\ldots+\mu_{n}^{*}$ is always a non-negative numerical measure.

\section{Definition 5.5}

A measurable set $E$ is an atom if $\mu(E) \neq 0$ and if for every measurable set $F \subset E$ either $\mu(F)=0$ or $\mu(F)=u(E)$. $u$ is non-atomic if none of its coordinates, $u_{i}(i=1, \ldots, n)$ has any atoms.

\section{Definition 5.6}

The measure $u$ is absolutely continuous with respect to the measure $v$, if

$$
\left|\nu^{*}(E)\right|=0 \text { implies }\left|u^{*}(E)\right|=0 \text { for any } E \in S \text {. }
$$

\section{Definition 5.7}

A measure $\mu$ is semi-convex if every measarable set $E$ contains a measurable set $F$ for which $u(F)=\mu(E) / 2$.

\section{Definition 5.8}

For any measure $u$ and any measurable set $E$ we define $K(\mu, E)$ to be the class of all real-valued measurable functions $\phi$ on $\mathbb{E}$ 
for which

(i) $0 \leqslant \phi(x)<1$ for all $x \in E$

and

(ii) $\mu(\{\mathrm{x} \in \mathrm{E}: \phi(\mathrm{x})\langle\lambda\})=\lambda \mu(E)$ for any $\lambda, 0 \leqslant \lambda \leqslant 1$.

Definition 5.9

A measure $\mu$ is convex if for every measurable set $E$, the class $K(\mu, E)$ is not empty.

Lemma 5.10

If $\mu$ is a semi-convex measure and $E$ is any measurable set then there exists a sequence $\left\{E_{n}\right\}_{n=1}^{\infty}$ of measurable subsets of $E$ such that for every $k=1,2, \ldots$ and any $k$ distinct positive integers $\mathrm{n}_{1}, \ldots, \mathrm{n}_{\mathrm{k}}$ we have $\mu\left(\mathrm{E}_{\mathrm{n}_{1}} \cap \ldots \cap \mathrm{E}_{\mathrm{n}_{\mathrm{k}}}\right)=\left(1 / 2^{k}\right) \mu(E)$.

\section{Lemma 5.11}

A semi-convex measure is convex.

Proof:

Let $u$ be a non-negative semi-convex measure. Let $E$ be any measurable set and let $\left\{E_{n}\right\}_{n=1}^{\infty}$ be a sequence of sets with the property defined in lemma 5.10.

If $x \in E_{*}=\lim _{n \rightarrow \infty} \inf _{n}$ then write $\phi(x)=0$; otherwise

write

$$
\phi(x)=\sum_{n=1}^{\infty} \epsilon_{n}(x) / 2^{n}
$$

where $\epsilon_{n}$ is the characteristic function of the set $E_{n}$. Clearly $\phi$ is measurable and $0 \leqslant \phi(x)<1$ for all $x \in E$. Since 


$$
\{x: \phi(x)<\lambda\}=E_{*} \cup\left\{x: \sum_{i=1}^{n} \epsilon_{n}(x) / 2^{i}<\lambda\right\}
$$

for any dyadically rational number $\lambda=k / 2^{n}$, with $n=0,1,2, \ldots$ and $k=1,2, \ldots, 2^{n}$; it follows that

$$
u(\{x: \phi(x)<\lambda\})=\lambda \mu(E)
$$

for all such values of $\lambda$. The fact that equality (5.1) is true for all $\lambda, 0 \leqslant \lambda \leqslant 1$, follows from the countable additivity of $\mu$. The truth of the lemma in the general case follows from the above by means of the device used in lemma 5.17.

Lemma 5.12

Let $u$ be a non-negative convex measure and let $E$ be any measurable set. If $\phi \in K(U, E)$ and if $v$ is a measure absolutely continuous with respect to $\mu$, then $v(\{x: \phi(x)<\lambda\})$ is a continuous function of $\lambda, \quad 0 \leqslant \lambda \leqslant 1$.

Lemma 5.13

If $\mu$ is a convex measure and $E$ and $F$ are any two measurable sets, then for each $\lambda, 0 \leqslant \lambda \leqslant 1$, there exists a measurable set $C(\lambda)$ with the following properties:

(i) $\begin{array}{ll}\mathrm{C}(0)=\mathrm{E} & \mathrm{C}(1)=\mathrm{F}\end{array}$

(ii) $\mu(C(\lambda))=(1-\lambda) \mu(E)+\lambda \mu(F)$

(iii) If $u$ is also non-negative and if $\nu$ is absolutely continuous with respect to $\mu$ then $v(C(\lambda))$ is a continuous function of $\lambda$.

Proof:

Let $\phi$ and $\psi^{\prime}$ be functions in $K(\mu, E-F)$ and $K(\mu, F-E)$ respectively; write 


$$
C(\lambda)=(E \cap F) \quad U \quad\{x: \phi(x)<1-\lambda\} \quad U \quad\{x: \psi(x)<\lambda\}
$$

Then (i) follows from the definition of $C(\lambda)$ and (iii) follows from lemma 5.12. (ii) is proved by the following:

$$
\begin{aligned}
u(C(\lambda)) & =u(E \cap F)+u(\{x: \phi(x)<1-\lambda\})+u(\{x: \psi(x)<\lambda\}) \\
& =u(E \cap F)+(1-\lambda) u(E-F)+\lambda u(F-E) \\
& =(1-\lambda) u(E)+\lambda u(F) .
\end{aligned}
$$

Corollary 5.14

The range of a convex measure is convex.

\section{Lemma 5.15}

If $u=\left(u_{1}, \ldots, u_{n}\right)$ is non-negative and non-atomic and if each $\mu_{i}(1<i \leqslant n)$ is absolutely continuous with respect to its predecessor, $u_{i-1}$, then $u$ is convex.

Proof:

Because of lemma 5.11, it suffices to prove that $\mu$ is semiconvex. In [3], lemmas 1 and 2, Halmos shows that the result holds when $n=1$. (It is shown that a non-negative, non-atomic, numerical measure defined on a orring of subsets of a measurable set $\mathrm{X}$, assumes a.l real values between 0 and $\mu(\mathrm{X})$. )

Suppose now (in the case $n>1$ ) that the $(n-1)$-dimensional measure $\mu^{\prime}=\left(u_{1}, \ldots, u_{n-1}\right)$ is convex. It follows from the hypotheses that the I-dimensional measure $v^{\prime}=\mu_{n}$ is absolutely continuous with respect to it.

Let $E \in S$. Then there exists a measurable set $E_{0} \subset E$ such that $\mu^{\prime}\left(E_{0}\right)=u^{\prime}(E) / 2$; write $E_{0}=E-E_{0}$. If $\nu^{\prime}\left(E_{0}\right)=\nu^{\prime}(E) / 2$ there is nothing to prove. Otherwise we may assume 


$$
v^{\prime}\left(E_{0}\right)<v^{\prime \prime}(E) / 2 \text { and } v^{\prime}\left(F_{0}\right)>v^{\prime \prime}(E) / 2
$$

Then Iemma 5.13 applied to $u^{\prime}, v^{\prime}, E_{0}, F_{0}$ in place of $u, i, E, F$ yields the existence of a number $\lambda, 0 \leqslant \lambda \leqslant 1$, such that

$$
\vartheta^{\prime}(C(\lambda))=v^{\prime}(E) / 2
$$

and

$$
\begin{aligned}
u^{\prime}(C(\lambda)) & =(I-\lambda) u^{\prime}\left(E_{0}\right)+\lambda u^{\prime}\left(F_{0}\right) \\
& =(I-\lambda) u^{\prime}(E) / 2+\lambda u^{\prime}(E) / 2=u^{\prime}(E) / 2 .
\end{aligned}
$$

Lemma 5.16

A non-atomic, non-negative vector measure is convex.

Proof:

If $u=\left(u_{1}, \ldots, u_{n}\right)$ is the measure in question, write

$$
\mu_{i}^{\prime}=\sum_{j=i}^{n} u_{j}, \quad i=1, \ldots, n .
$$

Then $u^{\prime}=\left(u_{1}^{\prime}, \ldots, u_{r 1}^{\prime}\right)$ satisfies the conditions of lemma 5.15 .

Therefore $u^{\prime}$ is convex.

Let $I$ be the inverse of the linear transformation which carries $u$ into $u^{\prime}$. Then the linearity of $I$ and the semi-convexity of $u^{\prime}$ imply that $u$ is semi-convex and hence convex.

\section{Lemma 5.17}

A non-atomic vector measure is convex.

Proof:

For each $i=1, \ldots, n$ there exists a measurable set $E_{1}$ such that $u_{i}\left(E \cap E_{i}\right) \geqslant 0$ and $u_{i}\left(E-E_{i}\right) \leqslant 0$ for every $E \in S$. (The Hahn decomposition with respect to each $u_{i}$ respectively.) 
On each of the measurable subsets of any one of the $2^{n}$ sets of the form $E_{1} \epsilon_{I} \cap \ldots \cap E_{n} \epsilon_{n} \quad\left(\epsilon_{i}=0\right.$ or $I \quad i=1, \ldots, n$ where $E_{j}{ }^{I}=E_{j}$ and $\left.E_{j}^{0}=X-E_{j}\right)$, each of the measures $u_{1}, \ldots, u_{n}$ is of constant sign. Lemma 5.16 may now be applied to the set function $\mu_{\epsilon_{1}}, \ldots, \epsilon_{n}=\left( \pm u_{1}, \ldots, \pm u_{n}\right)$, where the ambiguous sign is chosen in each case so that $\mu_{\epsilon_{1}}, \ldots, \epsilon_{n}$ is a non-negative measure. The convexity of $u_{\epsilon_{1}}, \ldots, \epsilon_{n}$ and the disjointness of any two distinct sets of the form $E_{I} \epsilon_{I} \cap \ldots \cap E_{n} \epsilon_{n}$ imply the convexity of $u$.

Corollary 5.18

The range of a non-atomic, bounded, vector measure is convex.

6. Range of a Non-Atomic Unbounded Vector Measure

Let $\mu$ be a strongly $\sigma$ finite unbounded vector measure as defined in paragraph four.

\section{Definition 6.1}

An unbounded vector measure, $k$, defined on a $\sigma$-ring $S$ is said to be non-atomic if

(i) $u$ is non-atomic on measurable sets of finite measure.

(ii) $\mu(E)=\alpha^{\infty}$ for $E \in S$ implies that there exists a measurable $F \subset E$ such that $0<\|, F(F)\|<\infty$.

Definition 6.2

Let $E$ be a subset of $T$, the compactification of $E^{n}$ defined in paragraph 2. Now $E$ can be written in the form $\left.E=E_{1}\right\} E_{2}$, where $E_{1} \subset E^{n}$ and $E_{2}$ consists solely of the infinite points of 
E. We say that $E$ is convex if:

(i) $\mathrm{E}_{1}$ is a convex subset of $\mathrm{E}^{\mathrm{n}}$.

and

(ii) if $\alpha^{\infty} \in E_{2}$ then there exists $t_{0}>0$ and a vector $\beta$ such that $(\alpha t+\beta) \in E$ for all $t \geqslant t_{0}$.

Lemma 6.3

Let $\mu$ be an unbounded vector measure which is strongly o-finite and non-atomic. Let $E$ and $F$ be any two measurable sets such that $\|\mu(E)\|<\infty$ and $\|\mu(F)\|<\infty$. Then for each $\lambda, 0 \leqslant \lambda \leqslant 1$, there exists a measurable set $G(\lambda)$ such that

$$
u(G(\lambda))=\lambda \mu(E)+(1-\lambda) u(F) \text {. }
$$

Proof:

$$
\|\mu(E)\|<\infty \text { and }\|\mu(F)\|<\infty \text { imply }\|\mu(E \cup F)\|<\infty \text {. }
$$

Consider the orring, $\bar{S}$, of all sets in $S$ which are contained in $(E \cup F)$. Define $\bar{u}$ on $\bar{S}$ by

$$
\bar{M}(G)=M(G) \text { for all } G \in \bar{S} \text {. }
$$

Then $\bar{u}$ is obviously countably additive.

$$
\text { Lemma } 3.4 \text { implies that }\|\bar{\mu}(G)\|<\infty \text { for all } G \in \bar{S} \text {. }
$$

Therefore the range of $\bar{\mu}$ is bounded; for otherwise there would exist a sequence, $\left\{E_{n}\right\}_{n=1}^{\infty}$, of disjoint $\bar{S}-$ measurable sets such that

$$
\left\|\sum_{\substack{n=1 \\ n}}^{N} \bar{u}\left(E_{n}\right)\right\| \longrightarrow \infty \text { as } N \longrightarrow \infty \text {. }
$$

Then $\left\|\bar{\mu}\left(\bigcup_{n=1}^{\infty} E_{n}\right)\right\|_{=\infty}$, contradicting (6.1).

Thus $\bar{u}$ is a bounded, non-atomic vector measure and Corollary 5.18 implies that $\bar{\mu}$ has convex range. Therefore for any $\lambda, 0 \leqslant \lambda \leqslant 1$, there exists an $\bar{S}-$ measurable set, $G(\lambda)$, such that 


$$
\bar{u}(G(\lambda))=\lambda \bar{\mu}(E)+(1-\lambda) \bar{\mu}(F) .
$$

However from the definition of $\bar{u}$ and $\bar{s}$ we have:

$$
u(G(\lambda))=\lambda(E)+(I-\lambda) u(F) .
$$

\section{Definition 6.4}

A half-cylinder on a set $S$ contained in $E^{n}$ is defined to be the set of all vectors of the form $(x+\alpha t)$, where $x \in S$, $t \geqslant 0$, and $\alpha$ is a fixed direction. An open half-cylinder on a set $S$ contained in $E^{n}$ is the interior of the set defined above.

\section{Lemma 6.5}

Let $A$ be an unbounded convex set in $E^{n}$. Then there exists some m-dimensional hyperplane, $(1 \leqslant m \leqslant n)$, containing $A$ such that A contains a non-trivial open half-cylinder in the m-dimensional space.

Proof:

(a) We first consider the case of $\mathrm{E}^{2}$. If $\mathrm{A}$ is one-dimensional (i.e. an unbounded line segment), the lemma is obviously true. Thus we may suppose that the points of $A$ are not collinear.

Since $A$ is unbounded there exists a sequence, $\left\{x_{n}\right\}$, of points of $A$ such that $\left\|x_{n}\right\| \longrightarrow \infty$. Consider the set $\bigcup_{n}\left\{x_{n} /\left\|x_{n}\right\|\right\}$ which is contained in $S^{I}$, the unit sphere in 2-space. Since $S^{l}$ is compact there exists a subsequence $\left\{x_{n_{k}}\right\}_{k=1}^{\infty}$ such that:

(i) $\left\|x_{n_{k}}\right\| \longrightarrow \infty$ as $k \longrightarrow \rightarrow$

and

$$
\text { (ii) } x_{n_{k}} /\left\|x_{n_{k}}\right\|-\cdots \rightarrow \alpha \text { as } k-\rightarrow \infty
$$

for some unit vector $\alpha$. 
Let $\mathrm{x}, \mathrm{y}$ be two points of $\mathrm{A}$ such that the straight line joining them does not have the direction $\alpha$. Let $\mathrm{H}$ be the open halfcylinder on the line segment (xy) which extends to infinity in direction $\alpha$.

Let $z \in H$. Join $x z, y z$. Suppose $x z$, yz make angles $\epsilon_{1}, \epsilon_{2}$ respectively with the direction vector $\alpha$. Let $C_{1}$ and $C_{2}$ be the two-dimensional cones with base points $\mathrm{x}$ and $\mathrm{y}$ respectively all of whose generators make angles of $\epsilon_{I}$ and $\epsilon_{\mathbb{Z}}$ respectively with the vector $\alpha$.

Now (6.2) and (6.3) imply that $C_{1}$ and $C_{2}$ each contain alI but finitely many of the $\left\{\mathrm{x}_{n_{\mathrm{k}}}\right\}_{\mathrm{k}}$. Therefore there certainly exists an integer $\mathrm{k}$ such that $\mathrm{x}_{\mathrm{n}_{\mathrm{k}}} \in\left(\mathrm{C}_{1} \cap \mathrm{C}_{2}\right), \quad \mathrm{x}_{\mathrm{n}_{\mathrm{k}}} \neq \mathrm{x}, \mathrm{y} \cdot$ Clearly $\mathrm{z}$ is contained in the triangle formed by $x_{n_{k}}, x$, and $y$. However $A$ is convex. Therefore $\mathrm{z} \in \mathrm{A}$ and $\mathrm{H}$ is contained in $\mathrm{A}$. (b) The n-dimensional case follows in an entirely analagous manner. Again we get a sequence of points of $A,\left\{x_{n}\right\}_{n}$, satisfying (6.2) and (6.3).

We consider the lowest dimensional hyperplane which contains A and take this to be our space. Let this space have dimension $m$. Since $A$ is not contained in any $(m-1)$-dimensional hyperplane, there exist $\mathrm{m}$ linearly independent points of $\mathrm{A}$; $\mathrm{y}_{\mathrm{I}}, \ldots, \mathrm{y}_{\mathrm{m}}$; such that the direction vector $\alpha$ does not lie in the $(m-1)$-dimensional hyperplane determined by these points. Let $H$ be the open halfcylinder on base the simplex determined by the $m$ vertices; $\mathrm{y}_{1}, \ldots, \mathrm{y}_{\mathrm{m}}$; and extending to infinity in the direction $\alpha$. We choose any $z$ in $H$ and again consider the lines $y_{1} z, \ldots, y_{m} z$ which make angles of 
$\epsilon_{1}, \ldots, \epsilon_{\mathrm{m}}$ respectively with the direction vector $\alpha$. Let $\mathrm{C}_{1}, \ldots, \mathrm{C}_{\mathrm{m}}$ be cones with base points $\mathrm{y}_{1}, \ldots, \mathrm{y}_{\mathrm{m}}$ respectively whose generators all make angles of $\epsilon_{I}, \ldots, \epsilon_{\mathrm{m}}$ respectively with $\alpha$.

As before there exists an integer $\mathrm{k}$ such that

$x_{k} \ll\left(C_{1} \cap \ldots \cap C_{m}\right)$. Then $z$ is contained in the simplex spanned by the points $x_{k}, y_{1}, \ldots, y_{m}$ and therefore $z \in A$ because of the convexity of A. Thus $\mathrm{H}$ is contained in A.

Theorem 6.5

The range of a non-atomic, unbounded vector measure is convex (in the sense of definition 6.2).

Proof:

Let the range of the measure, $\mu$ be expressed in the form $E=E_{I} \cup E_{2}$ as in definition 6.2. Then lemma 6.3 implies that $E_{I}$ is a convex subset of $\mathrm{E}^{\mathrm{n}}$.

Since $\mu$ is an unbounded vector measure, there exists a set $G \in S$ such that $\mu(G)=\alpha$ and thus $E_{Z}=\left\{\alpha^{\infty}\right\}$. Since $\mu$ is strongly $\sigma$-finite this implies that $\mathrm{E}_{I}$ is an unbounded convex subset of $\mathrm{E}^{\mathrm{n}}$. Now lemma 6.5 implies that $\mathrm{E}_{1}$ contains an open halfcylinder in some m-dimensional subspace of $E^{n}(m \geqslant 1)$ and thus, a fortiori, a half-line of the form

$$
\beta+\gamma t \text { for all } t \geqslant t_{0}\left(t_{0} \text { fixed }\right) \text {, and some } \beta, y \in E^{n} \text {. }
$$

However, since the range of an unbounded vector measure can only tend to infinity in one direction, namely $\alpha$, we have $\gamma=\alpha$, which gives the desired result. 


\section{A Counter-Example}

In [2], Halmos proves that the range of any bounded vector measure is closed. In this paragraph, it is shown by means of a counter-example that this conclusion is not necessarily true in the case of unbounded vector measures. For convenience we give the example in the complex plane.

Theorem 7.1

Let $u$ be Lebesgue measure on the real line. Then the range of the unbounded complex measure, $v$, given by

$$
\downarrow(E)=\int_{E \cap(1, \infty)} d u+i \int_{E \cap(1, \infty)}\left[1 /\left(1+t^{2}\right)\right] d u
$$

is not closed.

Note: The range of the measure, $l$, is indicated in the following diagram.

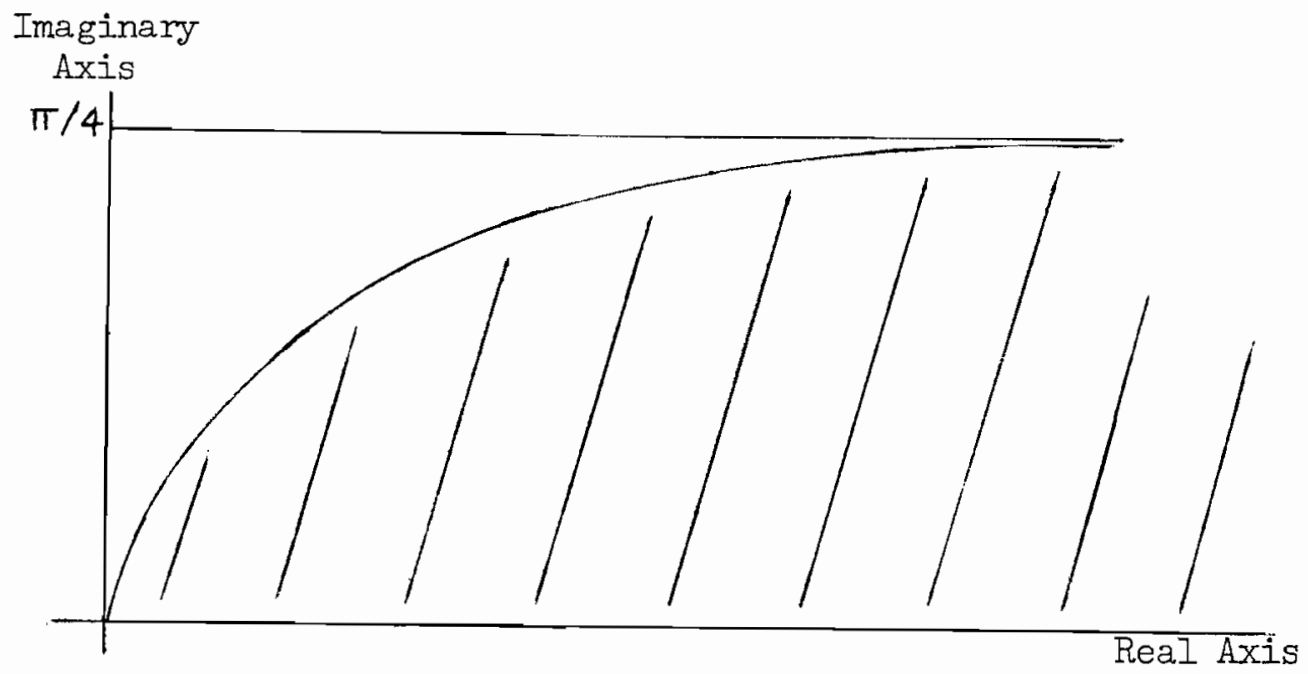


Proof:

$$
\int_{(1, \infty)}\left[1 /\left(1+t^{2}\right)\right] d u=\pi / 4<\infty
$$

and $\quad \int_{E \cap(1, \infty)} d \mu=u(E \cap(1, \infty)) \quad$ which can be made as large

as we please for appropriate sets $E$. Therefore $V(*)$ tends to infinity in the direction of the positive real axis.

$$
\text { Suppose } u(E \cap(I, \infty))>0 \text {. Since }\left[I /\left(I+t^{2}\right)\right]>0 \text { every- }
$$

where on $(I, \infty)$, therefore

$$
\int_{\operatorname{En}(1, \infty)}\left[1 /\left(1+t^{2}\right)\right] d \mu>0 .
$$

Thus $v($.$) assumes no values on the real axis other than the origin.$ Let $(x, 0), x>0$, be a given point on the real axis. Now $\mu[(n, n+x)]=x \quad n=1,2,3, \ldots$ and

$$
0<\int_{(n, n+x)}\left[1 /\left(1+t^{2}\right)\right] d u \leqslant\left[1 /\left(1+n^{2}\right)\right] x
$$

which can be made arbitrarily small for sufficiently large values of $n$. Thus $r($.$) assumes values as close to any point on$ the real axis as we please.

Therefore if $x>0$, the point $(x, 0)$ belongs to the closure of the range of $V($.$) , but is not in the range itself.$ Thus the range of $\gamma$ is not a closed set. 


\section{BIBLIOGRAPHY}

1. A. Liapounoff

Sur Ies fonctions-vecteurs completement additives,

Bull. Acad. Sci. URSS Ser. Math. vol. 4 (1940) 465-478

2. P.R. HaImos

The range of a vector measure,

BuII. A.M.S. vol. 54 (1948) 4I6-42I

3. P.R. Halmos

On the set of values of a finite measure,

Bull. A.M.S. VoI. 53 (1947) 138-14l (Iemmas 1 and 2)

(Note that the statement and proof of lemma 5 are wrong.)

4. P.R. Halmos

Measure Theory,

Princeton (1950)

5. H. Busemann

Convex Surfaces, (paragraph 1)

New York (1958)

6. G.G. Gould

Integration over vector-valued measures,

Proc. Ion. Math.Soc. vol. 15 part 11 (1965) 193-225 